\title{
On the Generation of Positivstellensatz Witnesses in Degenerate Cases*
}

\author{
David Monniaux ${ }^{\dagger} \quad$ Pierre Corbineau ${ }^{\ddagger}$
}

November 5, 2018

\begin{abstract}
One can reduce the problem of proving that a polynomial is nonnegative, or more generally of proving that a system of polynomial inequalities has no solutions, to finding polynomials that are sums of squares of polynomials and satisfy some linear equality (Positivstellensatz). This produces a witness for the desired property, from which it is reasonably easy to obtain a formal proof of the property suitable for a proof assistant such as Coq.

The problem of finding a witness reduces to a feasibility problem in semidefinite programming, for which there exist numerical solvers. Unfortunately, this problem is in general not strictly feasible, meaning the solution can be a convex set with empty interior, in which case the numerical optimization method fails. Previously published methods thus assumed strict feasibility; we propose a workaround for this difficulty.

We implemented our method and illustrate its use with examples, including extractions of proofs to Coq.
\end{abstract}

\section{Introduction}

Consider the following problem: given a conjunction of polynomial equalities, and (wide and strict) polynomial inequalities, with integer or rational coefficients, decide whether this conjunction is satisfiable over $\mathbb{R}$; that is, whether one can assign real values to the variables so that the conjunction holds. A particular case is showing that a given polynomial is nonnegative.

The decision problem for real polynomial inequalities can be reduced to quantifier elimination: given a formula $F$, whose atomic formulas are polynomial (in)equalities, containing quantifiers, provide another, equivalent, formula $F^{\prime}$, whose atomic formulas are still polynomial (in)equalities, containing no quantifier. An algorithm for quantifier elimination over the theory of real closed

*This work was partially supported by ANR project "ASOPT".

${ }^{\dagger}$ CNRS / VERIMAG

‡Université Joseph Fourier / VERIMAG 
fields (roughly speaking, $(\mathbb{R}, 0,1,+, \times, \leq)$ was first proposed by Tarski $[29,26]$, but this algorithm had non-elementary complexity and thus was impractical. Later, the cylindrical algebraic decomposition (CAD) algorithm was proposed [7], with a doubly exponential complexity, but despite improvements 8] CAD is still slow in practice and there are few implementations available.

Quantifier elimination is not the only decision method. Basu et al. [2, Theorem 3] proposed a satisfiability testing algorithm with complexity $s^{k+1} d^{O(k)}$, where $s$ is the number of distinct polynomials appearing in the formula, $d$ is their maximal degree, and $k$ is the number of variables. We know of no implementation of that algorithm. Tiwari 31] proposed an algorithm based on rewriting systems that is supposed to answer in reasonable time when a conjunction of polynomial inequalities has no solution.

Many of the algebraic algorithms are complex, which leads to complex implementations. This poses a methodology problem: can one trust their results? The use of computer programs for proving lemmas used in mathematical theorems was criticized in the case of Thomas Hales' proof of the Kepler conjecture. Similarly, the use of complex decision procedures (as in the proof assistant PVS1] or program analyzers (as, for instance, Astré 2 ) in order to prove the correctness of critical computer programs is criticized on grounds that these verification systems could themselves contain bugs.

One could formally prove correct the implementation of the decision procedure using a proof assistant such as Coq [30, 19]; but this is likely to be long and difficult. An alternative is to arrange for the procedure to provide a witness of its result. The answer of the procedure is correct if the witness is correct, and correctness of the witness can be checked by a much simpler procedure, which can be proved correct much more easily.

Unsatisfiability witnesses for systems of complex equalities or linear rational inequalities are already used within $\operatorname{DPLL}(T)$ satisfiability modulo theory decision procedures [16, ch. 11] [10]. It is therefore tempting to seek unsatisfiability witnesses for systems of polynomial inequalities.

In recent years, it was suggested [20] to use numerical semidefinite programming to look for proof witnesses whose existence is guaranteed by a Positivstellensatz [15, 28, 25]. The original problem of proving that a system of polynomial inequalities has no solution is reduced to: given polynomials $P_{i}$ and $R$, derived from those in the original inequalities, find polynomials $Q_{i}$ that are sums of squares such that $\sum_{i} P_{i} Q_{i}=R$. Assuming some bounds on the degrees of $Q_{i}$, this problem is in turn reduced to a semidefinite programming pure feasibility problem [6, 32], a form of convex optimization. The polynomials $Q_{i}$ then form a witness, from which a machine-checkable formal proof, suitable for tools such as Coq [30] or Isabelle [11], may be constructed.

Unfortunately, this method suffers from a caveat: it applies only under a strict feasibility condition [21]: a certain convex geometrical object should not be degenerate, that is, it should have nonempty interior. Unfortunately it is

\footnotetext{
${ }_{1}^{1}$ http://pvs.csl.sri.com/

${ }^{2}$ http://www.astree.ens.fr/
} 
very easy to obtain problems where this condition is not true. Equivalently, the method of rationalization of certificates [13] has a limiting requirement that the rationalized moment matrix remains positive semidefinite.

In this article, we explain how to work around the degeneracy problem: we propose a method to look for rational solutions to a general SDP feasibility problem. We have implemented our method and applied it to some examples from the literature on positive polynomials, and to examples that previously published techniques failed to process.

\section{Witnesses}

For many interesting theories, it is trivial to check that a given valuation of the variables satisfies a quantifier-free formula. A satisfiability decision procedure will in this case tend to seek a satisfiability witness and provide it to the user when giving a positive answer.

In contrast, if the answer is that the problem is not satisfiable, the user has to trust the output of the satisfiability testing algorithm, the informal meaning of which is "I looked carefully everywhere and did not find a solution." In some cases, it is possible to provide unsatisfiability witnesses: solutions to some form of dual or auxiliary problem that show that the original problem had no solution.

\subsection{Nonnegativity Witnesses}

To prove that a polynomial $P$ is nonnegative, one simple method is to express it as a sum of squares of polynomials. One good point is that the degree of the polynomials involved in this sum of squares can be bounded, and even that the choice of possible monomials is constrained by the Newton polytope of $P$, as seen in 83 .

Yet, there exist nonnegative polynomials that cannot be expressed as sums of squares, for instance this example due to Motzkin [23]:

$$
M=x_{1}^{6}+x_{2}^{4} x_{3}^{2}+x_{2}^{2} x_{3}^{4}-3 x_{1}^{2} x_{2}^{2} x_{3}^{2}
$$

However, Artin's answer to Hilbert's seventeenth problem is that any nonnegative polynomial can be expressed as a sum of squares of rational functions 3

It follows that such a polynomial can always be expressed as the quotient $Q_{2} / Q_{1}$ of two sums of squares of polynomials, which forms the nonnegativity witness, and can be obtained by solving $P . Q_{1}-Q_{2}=0$ for $Q_{1} \neq 0$ (this result is also a corollary of Th. 1).

\footnotetext{
${ }^{3}$ There exists a theoretical exact algorithm for computing such a decomposition for homogeneous polynomials of at most 3 variables [14]; we know of no implementation of it and no result about its practical usability.
} 


\subsection{Unsatisfiability Witnesses for Polynomial Inequalities}

For the sake of simplicity, we shall restrict ourselves to wide inequalities (the extension to mixed wide/strict inequalities is possible). Let us first remark that the problem of testing whether a set of wide inequalities with coefficients in a subfield $K$ of the real numbers is satisfiable over the real numbers is equivalent to the problem of testing whether a set of equalities with coefficients $K$ is satisfiable over the real numbers: for each inequality $P\left(x_{1}, \ldots, x_{m}\right) \geq 0$, replace it by $P\left(x_{1}, \ldots, x_{m}\right)-\mu^{2}=0$, where $\mu$ is a new variable. Strict inequalities can also be simulated as follows: $P_{i}\left(x_{1}, \ldots, x_{m}\right) \neq 0$ is replaced by $P_{i}\left(x_{1}, \ldots, x_{m}\right) . \mu=1$ where $\mu$ is a new variable. One therefore does not gain theoretical simplicity by restricting oneself to equalities.

Stengle 28] proved two theorems regarding the solution sets of systems of polynomial equalities and inequalities over the reals (or, more generally, over real closed fields): a Nullstellensatz and a Positivstellensatz; a similar result was proved by Krivine [15]. Without going into overly complex notations, let us state consequences of these theorems.

Let $K$ be an ordered field (such as $\mathbb{Q}$ ) and $K^{\prime}$ be a real closed field containing $K$ (such as the real field $\mathbb{R}$ ), and let $\mathbf{X}$ be a list of variables $X_{1}, \ldots, X_{n} . A^{* 2}$ denotes the squares of elements of $A$. The multiplicative monoid generated by $A$ is the set of products of zero of more elements from $A$. The ideal generated by $A$ is the set of sums of products of the form $P Q$ where $Q \in K[\mathbf{X}]$ and $P \in A$. The positive cone generated by $A$ is the set of sums of products of the form p.P. $Q^{2}$ where $p \in K, p>0, P$ is in the multiplicative monoid generated by $A$, and $Q \in K[\mathbf{X}]$. Remark that we can restrict $P$ to be in the set of products of elements of $A$ where no element is taken twice, with no loss of generality.

The result [18, 17, 9] of interest to us is:

Theorem 1. Let $F_{>}, F_{\geq}, F_{=}, F_{\neq}$be sets of polynomials in $K[\mathbf{X}]$, to which we impose respective sign conditions $>0, \geq 0,=0, \neq 0$. The resulting system is unsatisfiable over $K^{\prime n}$ if and only if there exist an equality in $K[\mathbf{X}]$ of the type $S+P+Z=0$, with $S$ in the multiplicative monoid generated by $F_{>} \cup F_{\neq}^{* 2}, P$ belongs to the positive cone generated by $F_{\geq} \cup F_{>}$, and $Z$ belongs to the ideal generated by $F_{=}$.

$(S, P, Z)$ then constitute a witness of the unsatisfiability of the system. 4

For a simple example, consider the following system, which obviously has no solution:

$$
\left\{\begin{array}{l}
-2+y^{2} \geq 0 \\
1-y^{4} \geq 0
\end{array}\right.
$$

A Positivstellensatz witness is $y^{2}\left(-2+y^{2}\right)+1\left(1-y^{4}\right)+2 y^{2}+1=0$. Another is $\left(\frac{2}{3}+\frac{y^{2}}{3}\right)\left(-2+y^{2}\right)+\frac{1}{3}\left(1-y^{4}\right)+1=0$.

Consider the conjunction $C: P_{1} \geq 0 \wedge \cdots \wedge P_{n} \geq 0$ where $P_{i} \in \mathbb{Q}\left[X_{1}, \ldots, X_{m}\right]$. Consider the set $\Pi$ of products of the form $\prod_{i} P_{i}^{w_{i}}$ for $\mathbf{w} \in\{0,1\}^{n}$ - that is,

\footnotetext{
${ }^{4}$ Another result, due to Schmüdgen [25], gives simpler witnesses for $P_{1} \geq 0 \wedge \cdots \wedge P_{n} \geq$ $0 \Rightarrow C$ in the case where $P_{1} \geq 0 \wedge \cdots \wedge P_{n} \geq 0$ defines a compact set.
} 
the set of all products of the $P_{i}$ where each $P_{i}$ appears at most once. Obviously, if one can exhibit nonnegative functions $Q_{j}$ such that $\sum_{T_{j} \in \Pi} Q_{j} T_{j}+1=0$, then $C$ does not have solutions. Theorem 1 guarantees that if $C$ has no solutions, then such functions $Q_{j}$ exist as sum of squares of polynomials (we simply apply the theorem with $F_{>}=F_{\neq}=\emptyset$ and thus $S=\{1\}$ ). We have again reduced our problem to the following problem: given polynomials $T_{j}$ and $R$, find sums-ofsquares polynomials $Q_{j}$ such that $\sum_{j} Q_{j} T_{j}=R$. Because of the high cost of enumerating all products of the form $\prod_{i} P_{i}^{w_{i}}$, we have first looked for witnesses of the form $\sum_{T_{j} \in S} Q_{j} P_{j}+1=0$.

\section{Solving the Sums-of-Squares Problem}

In $\$ 2.1$ and $\$ 2.2$, we have reduced our problems to: given polynomials $\left(P_{j}\right)_{1 \leq j \leq n}$ and $R$ in $\mathbb{Q}\left[X_{1}, \ldots, X_{m}\right]$, find polynomials that are sums of squares $Q_{j}$ such that

$$
\sum_{j} P_{j} Q_{j}=R
$$

We wish to output the $Q_{j}$ as $Q_{j}=\sum_{i=1}^{n_{j}} \alpha_{j i} L_{j i}^{2}$ where $\alpha_{j i} \in \mathbb{Q}^{+}$and $L_{j i}$ are polynomials over $\mathbb{Q}$. We now show how to solve this equation.

\subsection{Reduction to Semidefinite Programming}

Lemma 1. Let $P \in K[X, Y, \ldots]$ be a sum of squares of polynomials $\sum_{i} P_{i}^{2}$. Let $M=\left\{m_{1}, \ldots, m_{|M|}\right\}$ be a set such that each $P_{i}$ can be written as a linear combination of elements of $M$ ( $M$ can be for instance the set of monomials in the $\left.P_{i}\right)$. Then there exists a $|M| \times|M|$ symmetric positive semidefinite matrix $Q$ with coefficients in $K$ such that $P(X, Y, \ldots)=\left[m_{1}, \ldots, m_{|M|}\right] Q\left[m_{1}, \ldots, m_{|M|}\right]^{T}$, noting $v^{T}$ the transpose of $v$.

Assume that we know the $M_{j}$, but we do not know the matrices $\hat{Q}_{j}$. The equality $\sum_{j} P_{j}\left(M_{j} \hat{Q}_{j}\left(M_{j}\right)^{T}\right)=R$ directly translates into a system $(S)$ of affine linear equalities over the coefficients of the $\hat{Q}_{j}: \sum_{j}\left(M_{j} \hat{Q}_{j}\left(M_{j}\right)^{T}\right) P_{j}-R$ is the zero polynomial, so its coefficients, which are affine linear combinations of the coefficients of the $\hat{Q}_{j}$ matrices, should be zero; each of these combinations thus yields an affine linear equation. The additional requirement is that the $\hat{Q}_{j}$ are positive semidefinite.

One can equivalently express the problem by grouping these matrices into a block diagonal matrix $\hat{Q}$ and express the system $(S)$ of affine linear equations over the coefficients of $\hat{Q}$. By exact rational linear arithmetic, we can obtain a system of generators for the solution set of $(S): \hat{Q} \in-F_{0}+\operatorname{vect}\left(F_{1}, \ldots, F_{m}\right)$. The problem is then to find a positive semidefinite matrix within this search space; that is, find $\alpha_{1}, \ldots, \alpha_{m}$ such that $-F_{0}+\sum_{i} \alpha_{i} F_{i} \succeq 0$. This is the problem of semidefinite programming: finding a positive semidefinite matrix within an affine linear variety of symmetric matrices, optionally optimizing a linear form [32, 6]. 
For instance, the second unsatisfiability witness we gave for constraint system 2 is defined, using monomials $\{1, y\}, 1$ and $\{1, y\}$, by:

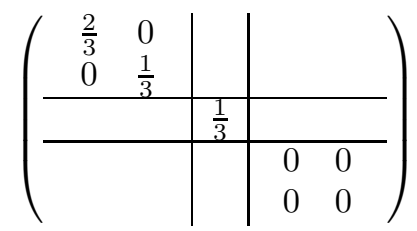

It looks like finding a solution to Equ. 3 just amounts to a SDP problem. There are, however, several problems to this approach:

1. For the general Positivstellensatz witness problem, the set of polynomials to consider is exponential in the number of inequalities.

2. Except for the simple problem of proving that a given polynomial is a sum of squares, we do not know the degree of the $Q_{j}$ in advance, so we cannot 5 choose finite sets of monomials $M_{j}$. The dimension of the vector space for $Q_{j}$ grows quadratically in $\left|M_{j}\right|$.

3. Some SDP algorithms can fail to converge if the problem is not strictly feasible - that is, the solution set has empty interior, or, equivalently, is not full dimensional (that is, it is included within a strict subspace of the search space).

4. SDP algorithms are implemented in floating-point. If the solution space is not full dimensional, they tend to provide solutions $\hat{Q}$ that are "almost" positive semidefinite (all eigenvalues greater than $-\epsilon$ for some small positive $\epsilon$ ), but not positive semidefinite.

Regarding problem 1, bounds on degrees only matter for the completeness of the refutation method: we are guaranteed to find the certificate if we look in a large enough space. They are not needed for soundness: if we find a correct certificate by looking in a portion of the huge search space, then that certificate is correct regardless. This means that we can limit the choice of monomials in $M_{j}$ and hope for the best.

Regarding the second and third problems : what is needed is a way to reduce the dimension of the search space, ideally up to the point that the solution set is full dimensional. As recalled by [21], in a sum-of-square decomposition of a polynomial $P$, only monomials $x_{1}^{\alpha_{1}} \ldots x_{n}^{\alpha_{n}}$ such that $2\left(\alpha_{1}, \ldots, \alpha_{n}\right)$ lies within the Newton polytop 6 of $P$ can appear [22, Th. 1]. This helps reduce the dimension if $P$ is known in advance (as in a sum-of-squares decomposition to prove positivity) but does not help for more general equations.

Kaltofen et al. [12] suggest solving the SDP problem numerically and looking for rows with very small values, which indicate useless monomials that can be

\footnotetext{
${ }^{5}$ There exist non-elementary bounds on the degree of the monomials needed [18]. In the case of Schmüdgen's result on compact sets, there are better bounds [25].

${ }^{6}$ The Newton polytope of a polynomial $P$, or in Reznick's terminology, its cage, is the convex hull of the vertices $\left(\alpha_{1}, \ldots, \alpha_{n}\right)$ such that $x_{1}^{\alpha_{1}} \ldots x_{n}^{\alpha_{n}}$ is a monomial of $P$.
} 
safely removed from the basis; in other words, they detect "approximate kernel vectors" from the canonical basis. Our method is somehow a generalization of theirs: we detect kernel vectors whether or not they are from the canonical basis.

In the next section, we shall investigate the fourth problem: how to deal with solution sets with empty interior.

\subsection{How to Deal with Degenerate Cases}

In the preceding section, we have shown how to reduce the problem of finding unsatisfiability witnesses to a SDP feasibility problem, but pointed out one crucial difficulty: the possible degeneracy of the solution set. In this section, we explain more about this difficulty and how to work around it.

Let $\mathcal{K}$ be the cone of positive semidefinite matrices. We denote by $M \succeq 0$ a positive semidefinite matrix $M$, by $M \succ 0$ a positive definite matrix $M$. The vector $\mathbf{y}$ is decomposed into its coordinates $y_{i}$. $\tilde{x}$ denotes a floating-point value close to an ideal real value $x$.

We consider a SDP feasibility problem: given a family of symmetric matrices $F_{0}, F_{i}, \ldots, F_{m}$, find $\left(y_{i}\right)_{1 \leq i \leq m}$ such that

$$
F(\mathbf{y})=-F_{0}+\sum_{i=1}^{m} y_{i} F_{i} \succeq 0 .
$$

The $F_{i}$ have rational coefficients, and we suppose that there is at least one rational solution for $\mathbf{y}$ such that $F(\mathbf{y}) \succeq 0$. The problem is how to find such a solution.

If nonempty, the solution set $S \subseteq \mathbb{R}^{m}$ for the $\mathbf{y}$, also known as the spectrahedron, is semialgebraic, convex and closed; its boundary consists in y defining singular positive semidefinite matrices, its interior are positive definite matrices. We say that the problem is strictly feasible if the solution set has nonempty interior. Equivalently, this means that the convex $S$ has dimension $m$.

Interior point methods used for semidefinite feasibility, when the solution set has nonempty interior, tend to find a solution $\tilde{\mathbf{y}}$ in the interior away from the boundary. Mathematically speaking, if $\tilde{\mathbf{y}}$ is a numerical solution in the interior of the solution set, then there is $\epsilon>0$ such that for any $\mathbf{y}$ such that $\|\mathbf{y}-\tilde{\mathbf{y}}\| \leq \epsilon$, $\mathbf{y}$ is also a solution. Choose a very close rational approximation $\mathbf{y}$ of $\tilde{\mathbf{y}}$, then unless we are unlucky (the problem is almost degenerate and all any suitable $\epsilon$ is extremely small), then $\mathbf{y}$ is also in the interior of $S$. Thus, $F(\mathbf{y})$ is a solution of problem 4 .

This is why earlier works on sums-of-square methods [21] have proposed finding rational solutions only when the SDP problem is strictly feasible. In this article, we explain how to do away with the strict feasibility clause.

Some problems are not strictly feasible. Geometrically, this means that the linear affine space $\left\{-F_{0}+\sum_{i=1}^{m} y_{i} F_{i} \mid\left(y_{1}, \ldots, y_{m}\right) \in \mathbb{R}^{m}\right\}$ is tangent to the semidefinite positive cone $\mathcal{K}$. Alternatively, this means that the solution set is included in a strict linear affine subspace of $\mathbb{R}^{m}$. Intuitively, this means that we 
are searching for the solution in "too large a space"; for instance, if $m=2$ and $\mathbf{y}$ lies in a plane, this happens if the solution set is a point or a segment of a line. In this case, some SDP algorithms may fail to converge if the problem is not strictly feasible, and those that converge, in general, will find a point slightly outside the solution set. The main contribution of this article is a workaround for this problem.

\subsection{Simplified algorithm}

We shall thus now suppose the problem has empty interior.

The following result is crucial but easily proved:

Lemma 2. Let $E$ be a linear affine subspace of the $n \times n$ symmetric matrices such that $E \cap \mathcal{K} \neq \emptyset$. F in the relative interior $I$ of $E \cap \mathcal{K}$. Then it follows:

1. For all $F^{\prime} \in E \cap \mathcal{K}$, $\operatorname{ker} F \subseteq \operatorname{ker} F^{\prime}$.

2. The least affine space containing $E \cap \mathcal{K}$ is $H=\{M \in E \mid \operatorname{ker} M \supseteq \operatorname{ker} F\}$.

Suppose we have found a numerical solution $\tilde{\mathbf{y}}$, but it is nearly singular meaning that it has some negative eigenvalues extremely close to zero. This means there is $\mathbf{v} \neq \mathbf{0}$ such that $|\mathbf{v} \cdot F(\tilde{\mathbf{y}})| \leq \epsilon\|\mathbf{v}\|$. Suppose that $\tilde{\mathbf{y}}$ is very close to a rational solution $\mathbf{y}$ and, that $\mathbf{v} \cdot F(\mathbf{y})=0$, and also that $\mathbf{y}$ is in the relative interior of $S$ - that is, the interior of that set relative to the least linear affine space containing $S$. Then, by lemma 2, all solutions $F\left(\mathbf{y}^{\prime}\right)$ also satisfy v. $F\left(\mathbf{y}^{\prime}\right)=0$. Remark that the same lemma implies that either there is no rational solution in the relative interior, or that rational solutions are dense in $S$.

How can finding such a $\mathbf{v}$ help us? Obviously, if $\mathbf{v} \in \bigcap_{i=0}^{m}$ ker $F_{i}$, its discovery does not provide any more information than already present in the linear affine system $-F_{0}+\operatorname{Vect}\left(F_{1}, \ldots, F_{m}\right)$. We thus need to look for a vector outside that intersection of kernels; then, knowing such a vector will enable us to reduce the dimension of the search space from $m$ to $m^{\prime}<m$.

Thus, we look for such a vector in the orthogonal complement of $\bigcap_{i=0}^{m} \operatorname{ker} F_{i}$, which is the vector space generated by the rows of the symmetric matrices $F_{0}, \ldots, F_{m}$. We therefore compute a full rank matrix $B$ whose rows span the exact same space; this can be achieved by echelonizing a matrix obtained by stacking $F_{0}, \ldots, F_{m}$. Then, $\mathbf{v}=\mathbf{w} B$ for some vector $\mathbf{w}$. We thus look for $\mathbf{w}$ such that $G(\mathbf{y}) \cdot \mathbf{w}=0$, with $G(\mathbf{y})=B F(\mathbf{y}) B^{T}$.

The question is how to find such a w with rational or, equivalently, integer coefficients. Another issue is that this vector should be "reasonable" - it should not involve extremely large coefficients, which would basically amplify the floating-point inaccuracies.

We can reformulate the problem as: find $\mathbf{w} \in \mathbb{Z}^{m} \backslash\{0\}$ such that both $\mathbf{w}$ and $G(\tilde{\mathbf{y}})$.w are "small", two constraints which can be combined into a single objective to be minimized $\alpha^{2}\|G(\tilde{\mathbf{y}}) \cdot \mathbf{w}\|_{2}^{2}+\|\mathbf{w}\|_{2}^{2}$, where $\alpha>0$ is a coefficient for tuning how much we penalize large values of $\|G(\tilde{\mathbf{y}}) \cdot \mathbf{w}\|_{2}$ in comparison to 
large values of $\|\mathbf{w}\|_{2}$. If $\alpha$ is large enough, the difference between $\alpha G(\tilde{\mathbf{y}})$ and its integer rounding $M$ is small. We currently choose $\alpha=\alpha_{0} /\|G(\tilde{\mathbf{y}})\|$, with $\|M\|$ the Frobenius norm of $M$ (the Euclidean norm for $n \times n$ matrices being considered as vectors in $\mathbb{R}^{n^{2}}$ ), and $\alpha_{0}=10^{15}$.

We therefore try searching for a small (with respect to the Euclidean norm) nonzero vector that is an integer linear combination of the $l_{i}=\left(0, \ldots, 1, \ldots, 0, m_{i}\right)$ where $m_{i}$ is the $i$-th row of $M$ and the 1 is at the $i$-th position. Note that, because of the diagonal of ones, the $l_{i}$ form a free family.

This problem is known as finding a short vector in an integer lattice, and can be solved by the Lenstra-Lenstra-Lovász (LLL) algorithm. This algorithm outputs a free family of vectors $s_{i}$ such that $s_{1}$ is very short. Other vectors in the family may also be very short.

Once we have such a small vector $\mathbf{w}$, using exact rational linear algebra, we can compute $F_{0}^{\prime}, \ldots, F_{m^{\prime}}^{\prime}$ such that

$$
\begin{aligned}
&\left\{-F_{0}^{\prime}+\sum_{i=1}^{m^{\prime}} y_{i}^{\prime} F_{i}^{\prime} \mid\left(y_{1}, \ldots, y_{m^{\prime}}\right) \in \mathbb{R}^{m^{\prime}}\right\}= \\
&\left\{-F_{0}+\sum_{i=1}^{m} y_{i} F_{i} \mid\left(y_{1}, \ldots, y_{m}\right) \in \mathbb{R}^{m}\right\} \cap\{F \mid F . \mathbf{v}=0\}
\end{aligned}
$$

The resulting system has lower search space dimension $m^{\prime}<m$, yet the same solution set dimension. By iterating the method, we eventually reach a search space dimension equal to the dimension of the solution set.

If we find no solution $F_{0}^{\prime}$, then it means that the original problem had no solution (the Positivstellensatz problem has no solution, or the monomial bases were too small), or that a bad vector $\mathbf{v}$ was chosen due to lack of numerical precision. This is the only bad possible outcome of our algorithm: it may fail to find a solution that actually exists; in our experience, this happens only on larger problems (search space of dimension 3000 and more), where the result is sensitive to numerical roundoff. In contrast, our algorithm may never provide a wrong result, since it checks for correctness in a final phase.

\subsection{More Efficient Algorithm}

In lieu of performing numerical SDP solving on $F=-F_{0}+\sum y_{i} F_{i} \succeq 0$, we can perform it in lower dimension on $-\left(B F_{0} B^{T}\right)+\sum y_{i}\left(B F_{i} B^{T}\right) \succeq 0$. Recall that the rows of $B$ span the orthogonal complement of $\bigcap_{i=0}^{m} \operatorname{ker} F_{i}$, which is necessarily included in ker $F$; we are therefore just leaving out dimensions that always provide null eigenvalues.

The reduction of the sums-of-squares problem (Eq. 3) provides matrices with a fixed block structure, one block for each $P_{j}$ : for a given problem all matrices $F_{0}, F_{1}, \ldots, F_{m}$ are block diagonal with respect to that structure. We therefore perform the test for positive semidefiniteness of the proposed $F(\mathbf{y})$ solution block-wise (see Sec. 3.6 for algorithms). For the blocks not found to be 
positive semidefinite, the corresponding blocks of the matrices $B$ and $F(\tilde{\mathbf{y}})$ are computed, and LLL is performed.

As described so far, only a single $\mathbf{v}$ kernel vector would be supplied by LLL for each block not found to be positive semidefinite. In practice, this tends to lead to too many iterations of the main loop: the dimension of the search space does not decrease quickly enough. We instead always take the first vector $\mathbf{v}^{(1)}$ of the LLL-reduced basis, then accept following vectors $\mathbf{v}^{(i)}$ if $\left\|\mathbf{v}^{(i)}\right\|_{1} \leq \beta .\left\|\mathbf{v}^{(1)}\right\|_{1}$ and $\left\|G(\tilde{\mathbf{y}}) \cdot \mathbf{v}^{(i)}\right\|_{2} \leq \gamma \cdot\left\|G(\tilde{\mathbf{y}}) \cdot \mathbf{v}^{(1)}\right\|_{2}$. For practical uses, we took $\beta=\gamma=10$.

When looking for the next iteration $\tilde{\mathbf{y}}^{\prime}$, we use the $\tilde{\mathbf{y}}$ from the previous iteration as a hint: instead of starting the SDP search from an arbitrary point, we start it near the solution found by the previous iteration. We perform leastsquare minimization so that $-F_{0}^{\prime}+\sum_{i=1}^{m^{\prime}} y_{i}^{\prime} F_{i}^{\prime}$ is the best approximation of $-F_{0}+\sum_{i=1}^{m} y_{i} F_{i} \mid\left(y_{1}, \ldots, y_{m}\right)$.

\subsection{Extensions and Alternative Implementation}

As seen in 4 , our algorithm tends to produce solutions with large numerators and denominators in the sum-of-square decomposition. We experimented with methods to get $F\left(\mathbf{y}^{\prime}\right) \approx F(\mathbf{y})$ such that $F\left(\mathbf{y}^{\prime}\right)$ has a smaller common denominator. This reduces to the following problem: given $\mathbf{v} \in \mathbf{f}_{0}+\operatorname{vect}\left(\mathbf{f}_{1}, \ldots, \mathbf{f}_{n}\right)$ a real (floating-point) vector and $\mathbf{f}_{0}, \ldots, \mathbf{f}_{n}$ rational vectors, find $\mathbf{y}_{1}^{\prime}, \ldots, \mathbf{y}_{n}$ such that $\mathbf{v}^{\prime}=\mathbf{f}_{0}+\sum_{i} y_{i}^{\prime} \mathbf{f}_{i} \approx \mathbf{v}$ and the numerators of $\mathbf{v}^{\prime}$ have a tunable magnitude (parameter $\mu$ ). One can obtain such a result by LLL reduction of the rows of:

$$
M=\left(\begin{array}{cccccc}
\mathbb{Z}\left(\beta \mu\left(\mathbf{f}_{0}-\mathbf{v}\right)\right) & \mathbb{Z}\left(\beta \mathbf{f}_{0}\right) & 1 & 0 & \ldots & 0 \\
\mathbb{Z}\left(\beta \mu \mathbf{f}_{1}\right) & \mathbb{Z}\left(\beta \mathbf{f}_{1}\right) & 0 & 1 & \ldots & \\
\vdots & \vdots & 0 & & \ddots & \\
\mathbb{Z}\left(\beta \mu \mathbf{f}_{n}\right) & \mathbb{Z}\left(\beta \mathbf{f}_{n}\right) & 0 & & & 1
\end{array}\right)
$$

where $\beta$ is a large parameter (say, $10^{19}$ ) and $\mathbb{Z}(v)$ stands for the integer rounding of $v$. After LLL reduction, one of the short vectors in the basis will be a combination $\sum_{i}^{n} y_{i} l_{i}$ where $l_{0}, \ldots, l_{n}$ are the rows of $M$, such that $y_{0} \neq 0$. Because of the large $\beta \mu$ coefficient, $y_{0}\left(\mathbf{f}_{0}-\mathbf{v}\right)+\sum_{i=1}^{n} y_{i} \mathbf{f}_{i}$ should be very small, thus $f_{0}+\sum_{i=1}^{n} y_{i} \mathbf{f}_{i} \approx v$. But among those vectors, the algorithm chooses one such that $\sum_{i=0}^{n} y_{i} \mathbf{f}_{i}$ is not large - and among the suitable $v^{\prime}$, the vector of numerators is proportional to $\sum_{i=0}^{n} y_{i} \mathbf{f}_{i}$.

After computing such a $y^{\prime}$, we check whether $F\left(\mathbf{y}^{\prime}\right) \succeq 0$; we try this for a geometrically increasing sequence of $\mu$ and stop as soon as we find a solution. The matrices $\hat{Q}_{j}$ then have simpler coefficients than the original ones. Unfortunately, it does not ensue that the sums of square decompositions of these matrices have small coefficients.

An alternative to finding some kernel vectors of a single matrix would be to compute several floating-point matrices, for instance obtained by SDP solving with optimization in multiple directions, and find common kernel vectors using LLL. 


\subsection{Sub-algorithms and Implementation}

The reduction from the problem expressed in Eq. 3 to SDP with rational solutions was implemented in Sage 7

Solving the systems of linear equations $(S)$ (Sec. 3.1 over the coefficients of the matrices) and 5 , in order to obtain a system $-F_{0}+\operatorname{vect}\left(F_{1}, \ldots, F_{m}\right)$ of generators of the solution space, is done by echelonizing the equation system (in homogeneous form) in exact arithmetic, then reading the solution off the echelon form. The dimension of the system is quadratic in the number of monomials (on the problems we experimented with, dimensions up to 7900 were found); thus efficient algorithms should be used. In particular, sparse Gaussian elimination in rational arithmetic, which we initially experimented, is not efficient enough; we thus instead use a sparse multi-modular algorithm [27, ch. 7] from LinBox 8 . Multi-modular methods compute the desired result modulo some prime numbers, and then reconstruct the exact rational values.

One can test whether a symmetric rational matrix $Q$ is positive semidefinite by attempting to convert it into its Gaussian decomposition, and fail once one detects a negative diagonal element, or a nonzero row with a zero diagonal element (Appendix. A). We however experimented with three other methods that perform better:

- Compute the minimal polynomial of $Q$ using a multi-modular algorithm [1]. The eigenvalues of $Q$ are its roots; one can test for the presence of negative roots using Descartes' rule of signs. Our experiments seem to show this is the fastest exact method.

- Compute the characteristic polynomial of $Q$ using a multimodular algorithm [1] and do as above. Somewhat slower but more efficient than Gaussian decomposition.

- Given a basis $B$ of the span of $Q$, compute the Cholesky decomposition of $B^{T} Q B$ by a numerical method. This decomposition fails if and only if $B^{T} Q B$ is not positive definite (up to numerical errors), thus succeeds if and only if $Q$ is positive semidefinite (up to numerical errors).

For efficiency, instead of computing the exact basis $B$ of the span of $Q$, we use $B$ from 33.3 , whose span includes the span of $Q$. The only risk is that $\operatorname{ker} B \subsetneq \operatorname{ker} Q$ while $Q$ is positive semidefinite, in which case $B^{T} Q B$ will have nontrivial nullspace and thus will be rejected by the Cholesky decomposition. This is not a problem in our algorithm: it just means that the procedure for finding kernel vectors by LLL will find vectors in $\operatorname{ker} Q \backslash \operatorname{ker} B$.

One problem could be that the Cholesky decomposition will incorrectly conclude that $B^{T} Q B$ is not positive definite, while it is but has very small

\footnotetext{
${ }^{7}$ Sage is a computer algebra system implemented using the Python programming language, available under the GNU GPL from http://www.sagemath.org

${ }^{8}$ LinBox is a library for exact linear arithmetic, used by Sage for certain operations. http://www.linalg.org/
} 
positive eigenvalues. In this case, our algorithm may then find kernel vectors that are not really kernel vectors, leading to an overconstrained system and possibly loss of completeness. We have not encountered such cases.

Another problem could be that a Cholesky decomposition is obtained from a matrix not positive semidefinite, due to extremely bad numerical behavior. At worst, this will lead to rejection of the witness when the allegedly semidefinite positive matrices get converted to sums of squares, at the end of the algorithm.

Numerical SDP solving is performed using $\operatorname{DSDP}^{9}$ [4, 3], communicating using text files. LLL reduction is performed by fpLLL 10 Least square projection is performed using Lapack's DGELS.

The implementation is available from the first author's Web page (http://bit. ly/fBNLhR and http://bit.1y/gPXNF8).

\subsection{Preliminary Reductions}

The more coefficients to find there are, the higher the dimension is, the longer computation times grow and the more likely numerical problems become. Thus, any cheap technique that reduces the search space is welcome.

If one looks for witnesses for problems involving only homogeneous polynomials, then one can look for witnesses built out of a homogeneous basis of monomials (this technique is implemented in our positivity checker).

One could also make use of symmetries inside the problem. For instance, if one looks for a nonnegativity witness $P=N / D$ of a polynomial $P$, and $P$ is symmetric (that is, there exists a substitution group $\Sigma$ for the variables of $P$ such that P. $\sigma=P$ for $\sigma \in \Sigma$ ), then one may reduce the search to symmetric $N$ and $D$. If $P=N / D$ is a witness, then $D P=N$ thus for any $\sigma,(D . \sigma) P=(N . \sigma)$ and thus $\left(\sum_{\sigma} D . \sigma\right) P=\left(\sum_{\sigma} N . \sigma\right)$, thus $D^{\prime}=\sum_{\sigma} D . \sigma$ and $N^{\prime}=\sum_{\sigma} N . \sigma$ constitute a symmetric nonnegativity witness.

\section{Examples}

The following system of inequalities has no solution (neither Redlog nor QepCad nor Mathematica 5 can prove it; Mathematica 7 can):

$$
\left\{\begin{array}{l}
P_{1}=x^{3}+x y+3 y^{2}+z+1 \geq 0 \\
P_{2}=5 z^{3}-2 y^{2}+x+2 \geq 0 \quad P_{3}=x^{2}+y-z \geq 0 \\
P_{4}=-5 x^{2} z^{3}-50 x y z^{3}-125 y^{2} z^{3}+2 x^{2} y^{2}+20 x y^{3}+50 y^{4}-2 x^{3} \\
\quad-10 x^{2} y-25 x y^{2}-15 z^{3}-4 x^{2}-21 x y-47 y^{2}-3 x-y-8 \geq 0
\end{array}\right.
$$

\footnotetext{
${ }^{9}$ DSDP is a sdp tool available from http://www.mcs.anl.gov/DSDP/

${ }^{10} \mathrm{fpLLL}$ is a LLL library from Damien Stehlé et al., available from http://perso.ens-lyon.fr/damien.stehle/
} 
$Q_{1}=8006878 A_{1}^{2}+29138091 A_{2}^{2}+25619868453870 / 4003439 A_{3}^{2}+14025608 A_{4}^{2}+14385502 A_{5}^{2}$

$+85108577038951965167 / 12809934226935 A_{6}^{2}$

$Q_{2}=8006878 B_{1}^{2}+25616453 B_{2}^{2}+108749058736871 / 4003439 B_{3}^{2}+161490847987681$

$/ 25616453 B_{4}^{2}+7272614 B_{5}^{2}+37419351 B_{6}^{2}+13078817768190 / 3636307 B_{7}^{2}+71344030945385471151$

$/ 15535579819553 B_{8}^{2}+539969700325922707586 / 161490847987681 B_{9}^{2}+41728880843834$

$/ 12473117 B_{10}^{2}+131008857208463018914 / 62593321265751 B_{1} 1^{2}$, where

$A_{1}=-1147341 / 4003439 x_{1}^{2} x_{3}-318460 / 4003439 x_{2}^{2} x_{3}+x_{3}^{3} A_{2}=x_{2} x_{3}^{2} A_{3}=-4216114037644$

$/ 12809934226935 x_{1}^{2} x_{3}+x_{2}^{2} x_{3} A_{4}=x_{1} x_{3}^{2}, A_{5}=x_{1} x_{2} x_{3}, A_{6}=x_{1}^{2} x_{3}$ and $B_{1}=-1102857$

$/ 4003439 x_{1}^{4} x_{2} x_{3}-5464251 / 4003439 x_{1}^{2} x_{2} x_{3}^{3}+2563669 / 4003439 x_{2}^{3} x_{3}^{3}+x_{2} x_{3}^{5}, B_{2}=-9223081$

$/ 25616453 x_{1}^{4} x_{3}^{2}-18326919 / 25616453 x_{1}^{2} x_{2}^{2} x_{3}^{2}+1933547 / 25616453 x_{2}^{4} x_{3}^{2}+x_{2}^{2} x_{3}^{4}$,

$B_{3}=-2617184886847 / 15535579819553 x_{1}^{4} x_{2} x_{3}-12918394932706 / 15535579819553 x_{1}^{2} x_{2} x_{3}^{3}+x_{2}^{3} x_{3}^{3}$

$B_{4}=-26028972147097 / 161490847987681 x_{1}^{4} x_{3}^{2}-135461875840584$

$/ 161490847987681 x_{1}^{2} x_{2}^{2} x_{3}^{2}+x_{2}^{4} x_{3}^{2}, B_{5}=-2333331 / 3636307 x_{1}^{3} x_{2} x_{3}^{2}-1302976$

$/ 3636307 x_{1} x_{2}^{3} x_{3}^{2}+x_{1} x_{2} x_{3}^{4}, B_{6}=-11582471 / 37419351 x_{1}^{5} x_{3}-12629854$

$/ 37419351 x_{1}^{3} x_{2}^{2} x_{3}-4402342 / 12473117 x_{1}^{3} x_{3}^{3}+x_{1} x_{2}^{2} x_{3}^{3}, B_{7}=-x_{1}^{3} x_{2} x_{3}^{2}+x_{1} x_{2}^{3} x_{3}^{2}$

$B_{8}=-x_{1}^{4} x_{2} x_{3}+x_{1}^{2} x_{2} x_{3}^{3}, B_{9}=-x_{1}^{4} x_{3}^{2}+x_{1}^{2} x_{2}^{2} x_{3}^{2}, B_{10}=-17362252580967 / 20864440421917 x_{1}^{5} x_{3}$

$-3502187840950 / 20864440421917 x_{1}^{3} x_{2}^{2} x_{3}+x_{1}^{3} x_{3}^{3}, B_{11}=-x_{1}^{5} x_{3}+x_{1}^{3} x_{2}^{2} x_{3}$.

Figure 1: Motzkin's polynomial $M$ (Eq. 1) as $Q_{2} / Q_{1}$.

This system was concocted by choosing $P_{1}, P_{2}, P_{3}$ somewhat haphazardly and then $P_{4}=-\left(P_{1}+\left(3+(x+5 y)^{2}\right) P_{2}+P_{3}+1+x^{2}\right)$, which guaranteed the system had no solution. The initial 130 constraints yield a search space of dimension 145 , and after four round of numeric solving one gets an unsatisfiability witness (sums of squares $Q_{j}$ such that $\sum_{j=1}^{4} P_{j} Q_{j}+Q_{5}=0$ ). Total computation time was $4.4 \mathrm{~s}$. Even though there existed a simple solution (note the above formula for $P_{4}$ ), our algorithm provided a lengthy one, with large coefficients (and thus unfit for inclusion here).

Motzkin's polynomial $M$ (Eq. 1) cannot be expressed as a sum of squares, but it can be expressed as a quotient of two sums of squares. We solved $M . Q_{1}-Q_{2}=0$ for sums of squares $Q_{1}$ and $Q_{2}$ built from homogeneous monomials of respective total degrees 3 and 6 - lesser degrees yield no solutions (Fig. 11). The equality relation over the polynomials yields 66 constraints over the matrix coefficients and a search space of dimension 186. Four cycles of SDP programming and LLL are then needed, total computation time was $4.1 \mathrm{~s}$.

We exhibited witnesses that each of the 8 semidefinite positive forms listed by [23], which are not sums of squares of polynomials, are quotients of sums of squares (Motzkin's $M$, Robinson's $R$ and $f$, Choi and Lam's $F, Q, S, H$ and Schmüdgen's $q$ ). These examples include polynomials with up to 6 variables and search spaces up to dimension 1155. We did likewise with delzell, laxlax and leepstarr2 from [12]. The maximal computation time was 7'.

We then converted these witnesses into Coq proofs of nonnegativity using a simple Sage script. These proofs use the Ring tactic, which checks for polynomial identity. Most proofs run within a few seconds, though laxlax takes 7'39" and Robinson's $f$ 5'07"; the witness for leepstarr2 is too large for the parser. We also exhibited a witness that the Vor1 polynomial cited by 24] is a sum of squares.

John Harrison kindly provided us with a collection of 14 problems that his system [1] could not find witnesses for. These problems generally have the 
form $P_{1} \geq 0 \wedge \cdots \wedge P_{n} \geq 0 \Rightarrow R \geq 0$. In order to prove such implication, we looked for witnesses consisting of sums of squares $\left(Q_{1}, \ldots, Q_{n}, Q_{R}\right)$,such that $\sum_{j} Q_{j} P_{j}+Q_{R} R=0$ with $Q_{R} \neq 0$, and thus $R=\frac{\sum_{j} Q_{j} P_{j}}{Q_{R}}$. In some cases, it was necessary to use the products $\prod_{i} P_{i}^{w_{i}}$ for $\mathbf{w} \in\{0,1\}^{n}$ instead of the $P_{i}$. We could find witnesses for all those problems 11. though for some of them, the witnesses are very large, taking up megabytes. Since these searches were done without making use of symmetries in the problem, it is possible that more clever techniques could find smaller witnesses.

\section{Conclusion and further works}

We have described a method for solving SDP problems in rational arithmetic. This method can be used to solve sums-of-squares problems even in geometrically degenerate cases. We illustrated this method with applications to proving the nonnegativity of polynomials, or the unsatisfiability of systems of polynomial (in) equalities. The method then provides easily checkable proof witnesses, in the sense that checking the witness only entails performing polynomial arithmetic and applying a few simple mathematical lemmas. We have implemented the conversion of nonnegativeness witnesses to Coq proofs. A more ambitious implementation, mapping Coq real arithmetic proofs goals to Positivstellensatz problems through the Psatz tactic from the MicroMega package [5], then mapping Positivstellensatz witnesses back to proofs, is underway.

One weakness of the method is that it tends to provide "unnatural" witnesses - they tend to have very large coefficients. These are machine-checkable but provide little insights to the reader. An alternative would be to provide the matrices and some additional data (such as their minimal polynomial) and have the checker verify that they are semidefinite positive; but this requires formally proving, once and for all, some non-trivial results on polynomials, symmetric matrices and eigenvalues (e.g. the Cayley-Hamilton theorem), as well as possibly performing costly computations, e.g. evaluating a matrix polynomial.

A more serious limitation for proofs of unsatisfiability is the very high cost of application of the Positivstellensatz. There is the exponential number of polynomials to consider, and the unknown number of monomials. It would be very interesting if there could be some simple results, similar to the Newton polytope approach, for reducing the dimension of the search space or the number of polynomials to consider. Another question is whether it is possible to define SDP problems from Positivstellensatz equations for which the spectrahedron has rational points only at its relative boundary.

While our method performed well on examples, and is guaranteed to provide a correct answer if it provides one, we have supplied no completeness proof that is, we have not proved that it necessarily provides a solution if there is one. This is due to the use of floating-point computations. One appreciable result would be that a solution should be found under the assumption that floating-

${ }^{11} \mathrm{~A} 7 \mathrm{z}$ archive is given at http://bit.1y/hM7HW3 
point computations are precise up to $\epsilon$, for a value of $\epsilon$ and the various scaling factors in the algorithm depending on the values in the problem or the solution.

It seems possible to combine our reduction method based on LLL with the Newton iterations suggested by [12, 13], as an improvement over their strategy for detection of useless monomials and reduction of the search space. Again, further experiment is needed.

\section{References}

[1] Jeffrey Adams, B. David Saunders and Zhendong Wan. "Signature of symmetric rational matrices and the unitary dual of Lie groups". in: $I S$ $S A C$ '05. Beijing, China: ACM, pages 13-20. ISBN: 1-59593-095-7. DOI: 10.1145/1073884.1073889

[2] Saugata Basu, Richard Pollack and Marie-Françoise Roy. "On the combinatorial and algebraic complexity of quantifier elimination". in: Journal of the ACM (JACM) 43.6 (), pages 1002-1045. DOI: http://doi.acm.org/10.1145/235809.235813

[3] Steven J. Benson and Yinyu Ye. "Algorithm 875: DSDP5 - Software for Semidefinite Programming" . in: ACM Transactions on Mathematical Software 34.3 (), 16:1-16-20. DOI: 10.1145/1356052.1356057.

[4] Steven J. Benson and Yinyu Ye. DSDP5 User Guide - Software for Semidefinite Programming. technical memorandum 277. Argonne National Laboratory,

[5] Frédéric Besson. "Fast Reflexive Arithmetic Tactics the Linear Case and Beyond". in: TYPES'06. LNCS 4502. Nottingham, UK: Springer, pages 48-62. ISBN: 3-540-74463-0. DOI: 10.1007/978-3-540-74464-1_4

[6] Stephen Boyd and Lieven Vandenberghe. Convex Optimization. Cambridge University Press, URL: http://www.stanford.edu/ boyd/cvxbook/.

[7] George E. Collins. "Quantifier elimination for real closed fields by cylindrical algebraic decomposition". in: Automata theory and formal languages. volume 33. LNCS. Springer, pages 134-183.

[8] Georges E. Collins. "Quantifier Elimination by Cylindrical Algebraic Decomposition - Twenty Years of Progress". in: Quantifier Elimination and Cylindrical Algebraic Decomposition. byeditor B. F. Caviness and J. R. Johnson. pages 8-23.

[9] Michel Coste, Henri Lombardi and Marie-Françoise Roy. "Dynamical method in algebra: effective Nullstellensätze". in: Annals of Pure and Applied Logic 111.3 (). Proceedings of the International Conference "Analyse \& Logique" Mons, Belgium 25-29 August 1997, pages 203-256. ISSN: 0168-0072. DOI: 10.1016/S0168-0072(01)00026-4.

[10] Bruno Dutertre and Leonardo de Moura. Integrating Simplex with $\operatorname{DPLL}(T)$. techreport SRI-CSL-06-01. SRI International, 
[11] John Harrison. "Verifying nonlinear real formulas via sums of squares". in: Proceedings of the 20th International Conference on Theorem Proving in Higher Order Logics, TPHOLs 200\% byeditor Klaus Schneider and Jens Brandt. volume 4732. LNCS. Springer, pages 102-118.

[12] Erich Kaltofen andothers. "Exact certification in global polynomial optimization via sums-of-squares of rational functions with rational coefficients". Accepted at J. Symbolic Computation.

[13] Erich Kaltofen andothers. "Exact certification of global optimality of approximate factorizations via rationalizing sums-of-squares with floating point scalars". in: ISSAC (International Symposium on Symbolic and Algebraic Computation). ACM, pages 155-163. DOI: 10.1145/1390768.1390792

[14] Etienne de Klerk and Dmitrii V. Pasechnik. "Products of positive forms, linear matrix inequalities, and Hilbert 17th problem for ternary forms". in: European Journal of Operational Research (), pages 39-45. DOI: $10.1016 / \mathrm{j}$.ejor.2003.08.014.

[15] Jean-Louis Krivine. "Anneaux préordonnés". in: J. Analyse Math. 12 (), pages 307-326. ISSN: 0021-7670. DOI: 10.1007/BF02807438, URL: http://hal.archives-ouvertes.fr/hal-00165658/en/.

[16] Daniel Kroening and Ofer Strichman. Decision procedures. Springer,

[17] Henri Lombardi. Théorème des zéros réels effectif et variantes. techreport. Besancon, France: Université de Franche-Comté, URL: http://hlombardi.free.fr/publis/ThZerMaj.pdf.

[18] Henri Lombardi. "Une borne sur les degrés pour le Théorème des zéros réel effectif". in: Real Algebraic Geometry: Proceedings of the Conference held in Rennes, France in 1991. byeditor Michel Coste, Louis Mahé and Marie-Françcoise Roy. volume 1524. Lecture Notes in Mathematics. Springer,

[19] Assia Mahboubi. "Implementing the CAD algorithm inside the Coq system". in: Mathematical Structures in Computer Sciences 17.1 (). DOI: doi:10.1017/S096012950600586X

[20] Pablo Parrilo. "Structured Semidefinite Programs and Semialgebraic Geometry Methods in Robustness and Optimization". phdthesis. California Institute of Technology,

[21] Helfried Peyrl and Pablo A. Parrilo. "Computing sum of squares decompositions with rational coefficients". in: Theoretical Computer Science 409.2 (), pages 269-281. DOI: 10.1016/j.tcs.2008.09.025.

[22] Bruce Reznick. "Extremal PSD forms with few terms". in: Duke Mathematical Journal 45.2 (), pages 363-374. DOI: 10.1215/S0012-7094-78-04519-2 
[23] Bruce Reznick. "Some Concrete Aspects Of Hilbert's 17th Problem". in: Real Algebraic Geometry and Ordered Structures. byeditor Charles N. Delzell and James J. Madden. volume 253. Contemporary Mathematics. American Mathematical Society,

[24] Mohab Safey El Din. "Computing the global optimum of a multivariate polynomial over the reals". in: ISSAC (International Symposium on Symbolic and Algebraic Computation). Linz/Hagenberg, Austria: ACM, pages 71-78. DOI: 10.1145/1390768.1390781.

[25] Markus Schweighofer. "On the complexity of Schmüdgen's Positivstellensatz". in: Journal of Complexity 20.4 (), pages 529-543. ISSN: 0885-064X. DOI: $10.1016 / \mathrm{j} \cdot \mathrm{jco} .2004 .01 .005$

[26] Abraham Seidenberg. "A New Decision Method for Elementary Algebra". in: Annals of Mathematics 60.2 (), pages 365-374. URL: http://www.jstor.org/stable/1969640

[27] William A. Stein. Modular forms, a computational approach. Graduate studies in mathematics. American Mathematical Society, ISBN: 978-08218-3960-7.

[28] Gilbert Stengle. "A Nullstellensatz and a Positivstellensatz in Semialgebraic Geometry". in: Mathematische Annalen 2.207 (), pages 87-07. DOI: 10.1007/BF01362149.

[29] Alfred Tarski. A Decision Method for Elementary Algebra and Geometry. University of California Press,

[30] The Coq Proof Assistant Reference Manual. 8.1. INRIA.

[31] A. Tiwari. "An Algebraic Approach for the Unsatisfiability of Nonlinear Constraints". in: Computer Science Logic, 14th Annual Conf., CSL 2005. byeditor L. Ong. volume 3634. LNCS. Springer, pages 248-262.

[32] Lieven Vandenberghe and Stephen Boyd. "Semidefinite Programming". in: SIAM Review 38.1 (), pages 49-95.

\section{A Gaussian Reduction and Positive Semidefi- niteness}

An algorithm for transforming a semidefinite positive matrix into a "sum of squares" form, also known as Gaussian reduction:

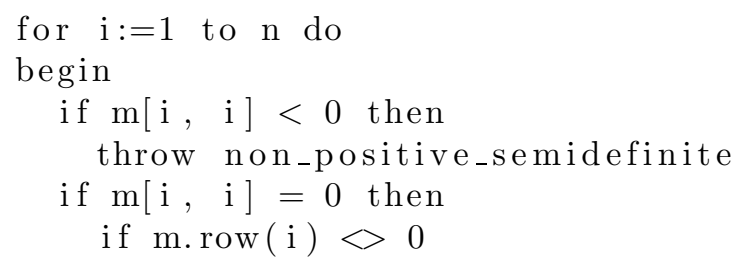




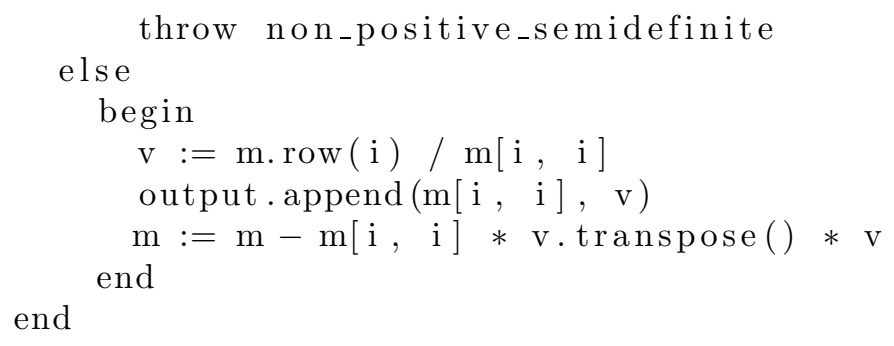

Suppose that the entrance of iteration $i, m[i \ldots n, i \ldots n]$ is positive semidefinite. If $m_{i, i}=0$, then the $i$ th base vector is in the isotropic cone of the matrix, thus of its kernel, and the row $i$ must be zero. Otherwise, $m_{i, i}>0$. By adding $\epsilon$ to the diagonal of the matrix, we would have a positive definite matrix and thus the output of the loop iteration would also be positive definite, as above. By $\epsilon \rightarrow 0$ and the fact that the set of positive semidefinite matrices is topologically closed, then the output of the loop iteration is also positive semidefinite.

The output variable is then a list of couples $\left(c_{i}, v_{i}\right)$ such that $c_{i}>0$ and the original matrix $m$ is equal to $\sum_{i} c_{i} v_{i}^{T} v_{i}$ (with $v_{i}$ row vectors). Otherwise said, for any row vector $u, u m u^{T}=\sum_{i} c_{i}\left\langle u, v_{i}\right\rangle^{2}$. 